[Aus dem Pathologischen Institut des städtischen Krankenhauses in Mainz (Vorstand: Georg B. Gruber).]

\title{
Zum Kapitel des peptischen Geschwürs im Kindesalter und der Melaena.
}

\author{
Von \\ Stephan Karl Mayer.
}

(Eingegangen am 4. August 1919.)

Die Erkenntnis vom Wesen des Magen- und Zwölffingerdarmgeschwürs hat mit der Vertiefung des Einblicks in seine morphologischen Verhältnisse nicht gleichen Schritt gehalten. Die Unsicherheit, woher man die Bedingungen seiner Entstehung ableiten soll, dokumentiert sich in der großen Zahl von Theorien, die auf die Lösung dieses Problems hinzielen. Sind doch ganz verschiedene, in ihrer Funktion charakteristische Gewebsanteile der Magenwand gesondert beschuldigt worden, im Zustand der Schädigung oder Minderwertigkeit die Grundlage zur Entwicklung eines Geschwürs abzugeben. Einmal. sollten die Epithelzellen zuviel Salzsäure, bald zu wenig Schleim, bald unzureichend Antipepsin bilden und damit einer Selbstverdauung Vorschub leisten, in der man den Grund des Geschwürs zu ersehen glaubte. Dann wurde in pathologischen Zuständen des Gefäßsystems, wie. Aneurysmen, Varicen oder Obliterationen und den daraus folgenden Iokalen Störungen der Blutverteilung, die primäre Schädigung. gesehen, welche durch Vermittlung einer Ischämie oder einer Gewebsblutung zu derartiger Ernährungsstörung der Mucosa führen sollte, daß daraus der geschwürige Affekt hergeleitet werden konnte. Schließlich wurde die Pathogenese des Ulcus ventriculi aus einer Disfunktion des Vagus oder Sympathicus, insonderheit aus lokalen Gefäß- oder Muskelkrämpfen abgeleitet. Damit nicht genug, wurden noch in letzter Linie außerhalb des Magens einsetzende Schädigungen, wie bakterielle Infektion oder Trauma, wiederum gesondert, zur Erklärung herangezogen.

Diese Anschauungen, auf die wir später zurückzukommen haben, sind oft auf großangelegten, statistischen Untersuchungen aufgebaut. 
Um so überraschender ist es, daß gerade die auf statistischen Ergebnissen beruhenden Theorien über das Ulcus pepticum durch neuere Forschungen nicht genügend bestätigt werden konnten. Man hat nun zwar frühzeitiger als bei anderen genetischen Krankheitsproblemen für div peptische Affektion erkannt, daß es mit einer Ursache hier nicht getan sein kann, und hat sich daran gewöhnt, einen Bedingungskomplex zu ergründen, der für die Entstehung von peptischen Geschwüren verantwortlich gemacht werden könnte. Auf die verschiedenste Weise ist man in der Forschung vorgegangen, die Ätiologie zu erklären, und hat dabei nur einzelne Bedingungsmöglichkeiten des Komplexes gefunden. Dies ist wohl der Grund, weshalb man, solange die Úberzeugung von der Multiplizität der Bedingungen fehlte, keine befriedigende Antwort auf die Entstehungsfrage des Ulcus pepticum geben konnte, wenigstens keine Antwort, welche dem Auftreten dieser Krankheit bei den verschiedenen Geschlechtern und in den verschiedenen Lebensaltern gerecht geworden wäre.

Noch zu Beginn unseres Jahrhunderts waren sich die meisten Autoren darüber einig, da $\beta$ das Ulcus pepticum in erster Linie Frauen befalle; ebenso glaubte man, daß es im frühen Kindesalter und im präsenilen bis senilen Alter so gut wie nicht vorkomme. Die Frauen sollen nach Josselin 5 mal, nach Meurell $3 \mathrm{mal}$ so oft als Männer an peptischem Magengeschwür erkranken. Leube gibt hierfür das Verhältnis 2:1 an. Diese Annahmen wurden namentlich in bezug auf Frauen von chirurgischer Seite zuerst stark in Frage gestellt. Heute herrscht Einigkeit darüber, daß Männer so oft wie Frauen davon befallen werden. So entnehmen wir einer Zusammenstellung G. B. Grubers, daß von 4208 Obduzierten 95 Männer, also $5 \%$, und 75 Frauen, also nur 4\%, peptische Affektion aufweisen.

Was die Anfälligkeit des Kindes für Ulcus pepticum betrifft, so sind die Angaben hierüber in einer so wertvollen und eingehenden Arbeit, wie sie die Rütimayersche Monographie darstellt, äußerst dürftig. Und noch 1912 wies Fischl ausdrücklich auf die ungemein große Seltenheit dieser Erkrankung sowohl für den Magen- als den Zwölffingerdarm bei Kindern hin. Aus den G. B. Gruberschen Feststellungen an Leichen geht hervor, daß von 1147 Kindern 17 peptisch affiziert waren, daß demnach diese Erkrankung nur $31 / 3$ mal $(1,5: 5 \%)$ seltoner bei Kindern als bei Erwachsenen auftritt. Collin fand sogar 20 Ulcera duodeni bei der Sektion von 237 Kindern im Alter von $1-10$ Jahren. Theile hat neuerdings 247 Ulcusfälle von Personen 
im Alter von 1-16 Jahren zusammengestellt, von denen 130 Kinder unter 1 Jahr aufgezählt sind. Man sieht also schon aus diesen Feststellungen, daß man dem kindlichen Ulcus ventriculi und duodeni ungleich häufiger begegnet, als dies nach früheren Angaben angenommen werden müßte. Diese Erfahrung legt den pathologischen Anatomen die besondere Aufgabe nahe, nach peptischen Affektionen auch dann zu fahnden, wenn von klinischer Seite kein Anhaltspunkt dafür gegeben ist. Denn der Kliniker kann nur durch Blutabgang per os oder per anum auf eine ulceröse Erkrankung hingewiesen werden, da ja sonst bei Kindern in der Regel keines der charakteristischen Anzeichen, wie Schmerz nach dem Essen oder Druckempfindlichkeit (Headsche Zone), zu erheben ist, zumal auch die Aciditätsbestimmungen, wenn sie überhaupt durchführbar sind, den Diagnostiker ganz im Stich lassen können.

ther das Vorkommen von peptischen Affektionen in präsenilen bis senilen Lebensalter liegen noch weniger brauchbare zahlenmäßige Unterlagen vor. Die höheren Häufigkeitsangaben sind nur dann verwertbar, wenn sie genau geschieden sind für noch vorhandene geschwürige Affekte und für Narben nach geschwürigen Affekten. Jedenfalls wiesen gerade Kirsch, Oberndorfer und $G$. B. Gruber auf eine überraschend große Beteiligung der höheren Lebensdezennien an den autoptischen Befunden dieser Art hin.

Die Durchsicht der einschlägigen Veröffentlichungen läßt weitere Beiträge wünschenswert erscheinen - und zwar gerade für die extremen Lebensdezennien des Greisenalters wie des Kindesalters. Aus dieser Tendenz heraus wurden in unserem Institut von Anfang an alle Kindesleichen einer eingehenden Prüfung hinsichtlich peptischer Affekte oder ihrer Vorstufen bzw. Residuen unterworfen. Die nachfolgenden Fälle fanden sich unter insgesamt 942 Sektionen von Kindern und Erwachsenen; sie sind die Ausbeute von 174 Kindersektionen des ersten Lebensdezenniums, welche innerhalb von 15 Monaten vorgenommen worden sind.

1. S.-Nr. 134/1917. J. Sch., Knabe, 3 Monate alt.

Klinische Diagnose: Atrophie, Herzschwäche, Darmkatarrh, kongenitale Lues.

Pathologisch-anatomische Diagnose: Pädatrophie. Bronchiolitis geringen Grades und geringer Ausdehnung. - Ulcus rotundum duodeni partis horizontalis. - Algemeine Anämie, Wassermannsche Reaktion an Leichenblut negativ. 
Das Ulcus war etwa linsengroß, sehr flach und etwas mißfarben gegenüber der graubräunlichen Farbe des übrigen Duodenums.

Mikroskopisch erwies sich das Uleus im Zwölffingerdarm als ein peptisches, bis an die Wandmuskulatur reichendes Duodenalgeschwür ohne erkennbare Arrosion der Gefäße. Die Bronchiolitis fand sich nur in den Randbezirken der Lungennterlappen. Nirgends Anzeichen für Lues.

2. S.-Nr. 283/1918. K. B., Mädehen, 3 Monate, 9 Tage alt.

Klinische Diagnose: Bronchitis.

Pathologisch-anatomische Diagnose: Eitrige Bronchitis in beiden Lungen mit kollaps-atelektatischen Herden. Duodenalgeschwürsna rbe knapp über der Papilla Vateri. Uleus auf der Zunge. Magenkatarrh. Schwellung der mesenterialen Lymphdrüsen.

Histologisch erwies sich der Befund einer strahligen Verdickung der Duodenalschleimhaut über der Papilla Vateri als eine von einschichtigem, zylindrischem Epithel bedeckte, etwas bindegewebighyperplastisch verdickte und gewulstete Submucosastelle, unter der die Wandmuskulatur ganz gewöhnlich verlief, während die Muscularis mucosa wie aufgesplittert und durchbrochen erschien. An den Grenzen der Unterbrechung war ein Teil der Muscularis-mucosae-Fasern gleichsam gegen die Mucosa hin abgelenkt.

Makroskopisch und mikroskopisch lag deshalb die Diagnose einer Narbenbildung nahe.

3. S.-Nr. 49/1918. S. H., Mädchen, 9 Jahre alt.

Klinische Diagnose: Meningitis tuberculosa.

Pathologiseh-a na to mische Diagnose: Allgemeine miliare Tuberkulose der Lungen, Milz, Leber, Nieren, ausgehend von einer käsigen bronchopneumonischen Infiltrations - Stelle der rechten Lungenspitze. Verkäsung der Lungenlymphdrüsen. Tuberkulöse Meningitis. Gallengangstuberkel in der Leber. Ältere solitäre Tuberkel in den Nieren. Geschwürige Darmtuberkulose, geschwürige Kehlkopftuberkulose. Käsige Tuberkulose der regionären Lymphdrüsen. Solitärer käsiger Tuberkel im Kleinhirn. Drei peptische Duodenalgeschwüre der Pars horizontalis und descendens. Innerer Wasserkopf. Ependymitis granularis.

Mikroskopisch: Die Ulcera duodeni erwiesen sich frei von Knötchenbildungen im Grund und im Randgebiet der Schleimhaut. Sie waren nicht durch Unterminierung der Mucosa ausgezeichnet. Die Muscularis mucosae stieg jederseits im Randgebiet des uleerösen Defektes steil nach oben und endete im nekrotischen Randbezirk. Blutgefäße waren nirgends arrodiert. 
4. S.-Nr. 314/1918. K. B., Knabe, 5 Tage alt.

Klinische Diagnose: Melaena.

Pathologisch-anatomische Diagnose: Absolute Ausblutung aus einem peptischen Geschwür im absteigenden Teil an der Rückwand des mittleren Teiles des Zwölffingerdarms; ein zweites, rundes Duodenalgeschwür an der Rückwand des horizontalen Teiles. Ein eröffnetes Blutgefäß war in Geschwürsbereich nicht zu sehen. Teerfarbener Inhalt in den unteren Darmabschnitten.

Mikroskopisch: Das Ulcus im absteigenden Teil erreichte die Muscularis propria. Die Muscularis mucosae war jederseits im Rand des Ulcusgebietes aufgekröpft. An einer Stelle fand sich ein arrodiertes kleines arterielles Gefä $\beta$, das von einem roten, etwas körnig aussehenden Gerinnselpfropf verstopft erschien.

5. S.-Nr. 437/1918. E. M., Mädchen, 6 Wochen alt.

Klinische Diagnose: Melaena.

Pathologisch-anatomische Diagnose: Multiple, peptisch hämorrhagische Erosionen der Magenschleimhaut. Gewebsmißbildung am Pförtner, leichter Pylorospasmus. Der als Gewebsmißbildung gedeutete Befund stellte sich als eine erbsengroße Vorwölbung im Schleimhautbereich des Magens $1 \mathrm{~cm}$ vor dem Pförtner dar. In der Mitte der Vorwölbung war eine kreisrunde, mundähnliche Vertiefung, die wie das Ende eines großen Ausführungsganges anzusehen war.

Mikroskopisch entpuppte sich diese Bildung als ein cylincirocelluläres Adenom der Magenwand, dessen wohlgeordnete Zẹltschläuche sich bis ins muskuläre und subseröse Gebiet erstreckten. - Die hämorrhagischen Erosionen ließen mikroskopisch einen Defekt der Epithelzellen erkennen. Darunter war das Gewebe oberflächlich nekrotisch, in Anschluß daran von ausgetretenem Blut durchsetzt, und zwar bis an die Muscularis propria hin.

6. S.-Nr. 505/1918. K. H., Mädchen, 2 Jahre alt.

Klinische Diag nose: Ruhr, Darmblutung (?).

Pathologisch-anatomische Diagnose: Geschwürige und verschorfende Dickdarmentzündung (Ruhr), peptisches Duodenalgeschwür mit schwerer Magen- und Darmblutung. (Das Geschwür war $2 \mathrm{~cm}$ über der Papilla, $1 \mathrm{~cm}$ lang, $1 / 2 \mathrm{~cm}$ breit.)

Das Ulcus des Duodenums wurde histologisch untersucht. Es zeigte einen Defekt der Mucosa, etwas unterminierten Rand pyloruswärts, während der Geschwürsgrund darmabwärts gegen den Geschwürsrand sich allmählich erhob. Der Grund des Geschwürs wurde von der 
Museularis gebildet; er war von lymphocytären Zellen, auch von Leukocyten durchsetzt. Auf den durchmusterten Schnitten wurde kein größeres Gefäß angetroffen, das von dem geschwürigen Prozeß angegriffen zu sein schien. Kleinste Gefäßchen waren zunächst dem Grund des Geschwürs mehrere zu sehen. Thre Wand ließ keine Verdickung oder Sklerose, ihr Lumen keine Thromben erkennen. Um sie herum waren Blutaustritte ins Gewebe feststellbar.

Es soll hier nun nicht die Lokalisation der gefundenen Ulcerationen näher besprochen werden. Dies ist für die Erwachsenen oft genug und bis jetzt, was die ätiologischen Folgerungsmöglichkeiten anlangt, in wenig fruchtbarer Weise geschehen. Auch wollen wir nicht auf das klinische Bild im ganzen eingehen, zumal es nur in recht spärlichen Zügen für obige Fälle niedergelegt worden ist; war doch in dem 1., 2. und 3. Fall der Befund einer peptischen Ulceration eine vollkommene Úberraschung und die Ulceration auch nicht die direkte Todesursache. Anders in den letzten 3 Fällen, von denen 2 sicherlich infolge der inneren Blutung ad exitum gekommen waren! Blutabgang war in diesen 3 Fällen mehr und minder vorhanden, so daß bei zweien in vivo die Diagnose Melaena gestellt werden konnte.

Das häufige Zusammentreffen von Melaena mit Ulcus pepticum beim Kind zwingt zur Überlegung, ob vielleicht für die Entstehung eines Ulcus wie für diejenige einer Melaena gemeinsame Gesichtspunkte bestehen. Daß dieser Gedanke nicht unmöglich ist, beweist schon die Utberlegung, daß recht häufig das Symptom der Melaena im Gefolge der peptischen Affektion auftritt; doch muß das ja nicht sein. Für beide Erscheinungen, Ulcus pepticum (bzw. hämorrhagisch-peptische Erosion) und Melaena gilt gemeinsam, daß die Magenwand, durch eine Schädigung ihres natürlichen Schutzes beraubt, der Verdauung durch den Magensaft anheimfällt, einerlei ob dieser natürliche Schutz allem lebenden Gewebe eigen ist oder eine besondere physiologische Anpassung der Magenschleimhaut an ihre Umgebung darstellt. Es besteht vielleicht auch die Möglichkeit, daß ein besonderes Antiferment normalerweise, $d . h$. bei ungestörten Zirkulationsbedingungen des Blutes, in der Magenwand zur Abwehr der Selbstverdauung gebildet wird. Jedenfatls stellt der Verlust der Resistenz der Magenwand gegenüber ihrem Sekret einen Faktor zur Entstehung von peptischen Affektionen dar. Die außerordentlich wichtigen anderen Komponenten, welche zur Bildung von Verdauungsdefekten der Magenwand nötig sind, werden dureh die Anwesenheit eines wirksamen Verdauungssaftes im Magen 
dargestellt. Das primär schädigende Moment zur Bildung eines peptischen Defektes wurde zunächst also in einem Versagen der lokalen Blutwirkung und Blutverteilung der Magenwand gesucht. Denn einmal wurde in der Alkalescenz des Blutes ein Schutz gegen Andaumg durch den sauren Magensaft, umgekehrt in der Zirkulationsstörung ein Verlust dieses Schutzes gesehen; dann war es auch einleuchtend, daß die Selbstaufzehrung des Magens am. leichtesten statthaben kann, wenn Abschluß von jeder Ernährung ein Weiterleben und Funktionieren der Schleimhaut an der betroffenen Stelle oder gar eine Regeneration unmöglich machte. Auch die späteren Theorien kommen letzten Endes auf eine Störung der Blutverteilung heraus; aber diese soll ihnen zufolge nicht direkt entstehen, durch lokale pathologische Vorgänge im Gefäßsystem selbst, wohin man ursprünglich den Sitz der Schädigung verlegt hatte (Lit. bei G. B. Gruber, Möller). Unter den häufig nach G. B. Gruber in $60 \%$ der Fälle - gleichzeitig mit peptischen Affektionen anzutreffenden Veränderungen des Herzens und der Gefäßwand spielt die Atheromatose die führende Rolle. Am meisten gefährdet sind durch ihre Folgen kleine Gefäße, wo der Mangel an Anastomosen eine besonders starke Reaktiou auf Kreislaufstörungen bedingt. Embolisation oder autochthon entstandene Thromben der Arteriolen führen zum Infarkt ihres Versorgungsgebietes, also der Submucosa und Mucosa umschriebener Zonen (Ha user). Besonders stark und oft kann nach G. B. Gruber die Abgangsstelle der Arteria coeliaea von atherosklerotischen Veränderungen befallen sein, wodurch die Zirkulation in allen aus ihr entspringenden Gefäßen gestört zu werden vermag.

Aber noch in anderem Sinne können Unregelmäßigkeiten des Blutkreislaufes zur Entstehung einer peptischen Affektion beitragen. Die peptischen Ulcera, die sich nach Bauchoperationen mit starken In. sulten, Bewegungen und Verlagerungen des Netzes einstellen können, sind durch embolische Störungen der venösen Blutversorgung der Magenwand erklärt worden, wobei die Vorstellung von der retrograden Embolie aus dem Pfortaderstamm in die Magenvenen eine Rolle spielt (Payr).

Es lassen aber diese Theorien durchaus nicht so klar den Zusammenhang von Ulcus und GefäB- und Zirkulationsschaden erkennen, als wünschenswert wäre. In so vielen Fällen fehlt noch jede pathologischanatomische Handhabe zur Anwendung der einen oder anderen Theorie von der Wichtigkeit der Mitwirkung hämodynamischer Störungen 
zum Zustandekommen einer peptischen Affektion. Und in anderen Fällen ersieht man aus dem recht häufigen Nebenbefund einer Myokarditis, einer Coronarsklerose, einer aneurysmatischen Aortenwanderkrankung, einer Stauungsleber usw. lediglich das eine, daß eine die Blutzirkulation beeinträchtigende Erscheinung neben dem Uleus pepticum zwar zu erkennen ist, weiß aber nicht, wie weit diese Erscheinung im Bedingungskomplex des peptischen Affektes in den Vordergrund geschoben werden muß. Die- ursächlichen Brücken, die man hier so gern sehen möchte, fehlen oder sind unsicher und recht nebelhaft; je weiter natürlich die räumliche Entfernung bestimmt lokalisierter Schädigungen im Gefäß- und Gefäßanastomosenbereich des Magendarmtraktus vom Ort des bei der Obduktion gesehenen Uleus pepticum ist, desto schwerer wird es sein, solche Brücken des Zusammenhangs zu konstruieren.

Wenn nun aber die Distanz so groß ist, daß ganz andere Organe, andere Körperteile' von einer Krankheitsschädigung betroffen wurden, und zugleich mit deren Folgen ein peptischer Affekt aufgespürt wurde, gibt es dann noch eine Möglichkeit, an den Zusammenhang der peptischen Defektbildung mit der weit entfernten Schädigung des anderen Organes oder Organsystems, z. B. von Gehirnabschnitten wie in unserem 3. Fall, zu denken? Es ist gelungen, nach Verletzung der Vierhügel, der Pedunculi cerebelli, des Thalamus usw. bei Versuchstieren, Ulcera peptica autoptisch festzustellen. Diese Versuche brachten den Gedanken nahe, daß in einer Vermittlung von Reizen durch das Nervensystem die Ursache für das Magengeschwür zu sehen sei. Bekanntlich führten systematische, experimentelle Untersuchungen von Talma, Lichtenbelt $u$. a., die indes nicht unwidersprochen blieben, zu der Annahme, daß eine Uberfunktion des Vagus einen Krampf der Muscularis mucosae auslöse, wodurch eine Ischämie der Magenwand und damit die Hauptbedingung für ein Ulcus pepticum veranlaßt würde. Von diesem Standpunkt ist zur Auffassung, daß überhaupt Gefäßkrämpfe die Ursache für die peptischen Affektionen abgeben können, nur ein Schritt, zumal wenn man bedenkt, daß ja längst dem Gefäßsystem eine ausschlaggebende, allerdings viel lokaler beschränkte Rolle zugedacht war. Durch Forschungen Rössles, G. v. Bergmanns und seiner Schule ist die Anschauung von der vasoneurotischen Natur der peptischen Prozesse näher beleuchtet und ausgebaut worden. Rössle hat an Hand von Statistiken dargelegt, daß peptische Affektionen oft als ,zweite Krankheit" nach Peritoneal- 
reizung, wie sie Appendicitis und auch Bauchoperationen mit sich bringen, und ebenso nach Erkrankungen im Kopf- und Halsbereich und anderem mehr, auftreten. Die vermittelnde Rolle zwischen der primären Schädigung und dem Defekt der Magenschleimhaut wurde dem Vagus zugeteilt, der bei dem betroffenen Patienten als übererregbar (Vagotonie) angenommen wurde. Den Beweis dafür glaubt Bergmann u. a. ex juvantibus mit den Erfolgen der Atropintherapie bei Ulcuskranken zu bringen.

Man kann, wenn man gewöhnt war, sich in Anschauungen über Krankheitsentstehung vom lokalistischen, pathologisch-anatomischen Prinzip leiten zu lassen, zunächst natürlich sich des Eindruckes nicht erwehren, daß solche neurogenen Theorien etwas Gesuchtes an sich haben. Wenn aber alles Augenfällige nicht zur Erklärung zu dienen vermag, muß man eben suchen, muß man wagen, durch kompliziertere Gedankengänge eine Vorstellung von rätselhaftem Geschehen zu erhalten. Und wenn man nach einer plausiblen Erklärung für die kindlichen peptischen Affektionen sucht, kommt man leicht auf solche Gedankengänge. Denn die Arteriosklerose, ebenso wie die anderen Gefäßaffektionen, etwa Aneurysma oder Intimaulcerationen, können bei Kindern als ätiologische Faktoren für Magen- und Zwölffingerdarmgeschwüre nicht in Betracht kommen; andererseits haben wir bei dem im Kindesalter öfters angetroffenen Pylorospasmus ein Beispiel dafür, daß der kindliche Magen im besonderen Maße von nervösen Einflüssen abhängig ist, wie ja ganz zweifellos reflektorische Krampfzustände im Kindesalter für den Arzt eine ganz alltägliche Beobachtung sind.

Einen direkten anatomischen Beweis für die Richtigkeit der neurogenen Theorien der peptischen Ulcusgenese zu liefern, wird unmöglich sein. Ein Nerv, durch welchen ein Reflex ablief, ist ja histologisch nicht geschädigt. Wir bleiben deshalb vorläufig auf die Beobachtung und Ausdeutung pathologisch-physiologischer Analoga im Versuch oder am Lebenden beschränkt. Wir müssen die Erscheinungsvorgänge an Hand genauester klinischer Beobachtung und pathologischer Postmortem-Untersuchung der möglicherweise geschaffenen Organveränderungen zueinander in Beziehung zu bringen suchen. Je eindeutiger die Erscheinungen im Leben waren, d. h. je weniger Organsysteme an den Erscheinungen sich beteiligten, je kürzer die Zeit zwischen diesen augenfälligen Erscheinungen und dem Tod war, und je prägnanter der pathologisch-anatomische Befund in dem betroffenen Organsystem sich darstellt, in welchem reflektorische Wellen der klinisch 
gegebenen Erscheinungen ablaufen konnten, desto eher wird unser Schluß der Abhängigkeit des einen Krankheitsgeschehens vom anderen Anspruch auf Richtigkeit haben.

In diesem Sinne sei ein Fall G. B. Grubers von Herzmuskelnekrose angeführt, dem die bei der neurogenen Genese des Ulcus pepticum sich abspielenden Vorgänge zum wenigsten sehr ähnlich sein mögen. „Es handelte sich um einen früher stets gesunden Epileptiker, der als solcher beim Militär entdeckt wurde und in der Aura der wenigen Anfälle, die er überhaupt hatte, die subjektiven Symptome der Angina pectoris bot. In einem echten Anfall ging der Mann zugrunde, nachdem er, mehrere Tage von stenokardischen Beschwerden und Ängsten gequält, das Kommen des Anfalls vorausgesagt. Die Obduktion ergab einen kräftigen, anatomisch durchaus gesunden Mann, der lediglich eine ganz frische Nekrose des Herzmuskels im Gebiet der vorderen Art. coronaria descendens zeigte. Dabei waren die Coronarien zart, glatt und völlig durchgängig wie die großen und kleinen sonstigen Schlagadern. Nirgends waren Thromben, nirgends sonstige Emboliequellen, nirgends steckten Emboli. Es blieb nur die Annahme übrig, daß hier ein Herzarterienkrampf, bedingt durch zentrale Einflüsse, wohl angioneurotisch vermittelt, AnlaB zum schweren irreparablen Herzschaden gegeben." Wenn solche Nekrosen auf offensichtlich neurovasculärer Basis im Herzen entstehen können, dann hat die Annahme gleicher Vỡgänge im Magen-Darmtraktus nichts Befremdendes mehr.

Ist nun die Auffassung der peptischen Affektionen bei Kindern als Folge einer neurogenen Schädigung zulässig, so wäre zu prüfen, ob sie auch einen Krankheitszustand erklärt, der oft mit Uleus kombiniert ist und für dessen Auslegung nicht wèniger Theorien aufgestellt sind - nämlich die Melaena neonatorum.

Vorausgeschickt sein mag hier eine allgemeine Bemerkung: Die Wandlung des Begriffes Melaena in der Medizin gibt ein gutes Bild davon, wie in der Heilkunde oft dasjenige als wesentlich an einem krankhaften Zustande angesehen wurde, was diesen Zustand gerade besonders bequem in die herrschenden Grundvorstellungen einreihte; oder praktischer ausgedrückt: Stets erscheint die Theorie am fruchtbarsten, die uns auf einen Weg weist, auf dem wir hoffen, jetzt oder später bestimmt das Ubel an der Wurzel zu packen. Auch Theile hat dies empfunden und darüber bemerkt: ,Beim Durchgehen der Geschichte der Melaena ist es interessant festzustellen, wie der Geist 
und die Ideen, welche jemals die betreffende Zeitperiode beherrschten, sich in der Auffassung und den Hypothesen widerspiegelt." Man weiß natürlich, daß das für alle Begriffe in der Medizin gilt, wenn es auch für den Melaenabegriff ganz besonders ausgeprägt ist.

So wurde im 18. Jahrhundert - einer Epoche, die Lique ux mit schlecht angebrachter Utberhebung die Période de confusion nannte entsprechend der damaligen Forschungsmethode unter Melaena jede Blutung ohne Unterschied ihres Herkommens bei Neugeborenen verstanden; denn man ging in der Medizin wie in der ganzen Naturwissenschaft - es sei nur an die Botanik erinnert - darauf aus, die Vorgänge nach der Mannigfaltigkeit ihrer äußeren Erscheinungen zu sondern, ihre Symptomatik festzulegen.

Als man später unter dem Einfluß von J. B. Morgagnis großartigem Werk über die Sedes morborum die Krankheiten auf ihre anatomischen Grundlagen prüfte und diese und jene klinisch wahrgenommene Affektion in bestimmte Organe oder Organteile lokalisierte, ließ man nur noch die aus dem Verdauungstraktus stammenden Blutungen als Melaena gelten. Damit schieden von den alten Beobachtungen einmal diejenigen aus, bei denen das Blut im Munde der Neugeborenen von der Mamma oder der Vagina der Mutter stammte oder durch Schluckbewegungen unter der Geburt etwa aspiriert war. Da konnten auch die Fälle nicht mehr als Melaena gelten, denen Blutungen der Neugeborenen aus der Nasen- und Paukenhöhle oder aber eine Metrorrhagie der neugeborenen Mädchen zugrunde lagen. Diese Fälle wurden als Melaena spuria bezeichnet.

Wie immer wurde, als einmal skeptisehe Unterscheidung und Kritik waltete, auch über das Ziel hinaus geschossen. S woboda ging in seiner Skepsis so weit, alle früheren Beobachtungen, bei denen von Bluterbrechen berichtet war, als aus dem Nasenrachenraum stammend anzusehen und das Vorkommen einer "Melaena vera" überhaupt zu leugnen. So hatte man nämlich die aus dem Magendarmkanal stammenden Blutungen von körpereigenem Blut beim Kinde genannt, ohne sich daran zu kehren, daß Melaena vom griechischen "Melas" abstammt und da $B$ die Bezeichnung ,Melaena vera" = ,wahrhaft dunkle Krankheit" für einen Zustand, dessen kausale und formale Pathogenese damals gänzlich ungeklärt war, ein selbstironisches Bekenntnis absoluter U'uzulänglichkeit ärztlichen Forschens enthielt.

In der darauf anbrechenden bakteriologischen Ära glaubte man ( (iärtner u. a.) wie für jede Krankheit, so auch für die Melaena einen 
spezifischen Erreger finden zu müssen, was bisher aber noch nicht gelungen ist. Abermals eine neue Periode, unter deren Einfluß wir heute noch stehen, geht darauf aus, verschiedene uns bisher klinisch als geschlossene Krankheitsbilder imponierende Erscheinungen im Sinne von Symptomen auf eine gemeinsame Affektion oder Minderwertigkeit eines ganzen Organsystems zurückzuführen. So teilt eben die Melaena das Schicksal mancher bisher seltener Krankheitsbilder; sie sank zur einzelnen Ausdrucksform für die pathologische Funktion eines Organsystems herab, sie wurde, etwa wie die Purpura, als Zeichen der hämorrhagischen Diathese aufgefaßt, einerlei ob sie mit greifbaren anatomischen, lokalisierten Veränderungen oder ohne solche auftritt, als ,symptomaticas. oder ,idiopathica", wie man früher auch gesagt hat. Denn selbst dann bleiben noch Fälle übrig, die nicht ohne weiteres erklärbar sein müssen, wie diejenigen, bei welchen scheinbar gänzlich ,,idiopathische“ Blutungen der Intestinalschleimhant (Vassmer, Heim) einen Blutabgang per os oder per anum veranlassen. Die Ursachen können in Eigenschaften des Blutes und der Gefäßwand und der lokalen Intestinalfunktion, des Vasomotorenapparates gelegen sein. Wir wissen es nicht und müssen von Fall zu Fall die formale wie kausale Genese zu ergründen suchen.

Hier sei eine Beobachtung mitgeteilt, welche als Beispiel dienen kann dafür, daß Varicen der Speiseröhre zur Blutungsquelle werden. Der Grund für die Varicose ist allerdings nicht ersichtlich. Vielleicht war sie in Verhältnissen des fötalen Blutkreislaufes begründet.

7. S.-Nr. 265/1918. J. S., Mädchen, 15 Tage alt.

Klinische Diagnose: Melaena neonatorum? Seit der Geburt Erbrechen und dünner Stuhl, beides Blut enthaltend. Nach Aufnahme ins Krankenhaus ebenfalls kaffeesatzartiges Erbrechen und schwarzer Stuhl. Kochsalzinfusionen.

Pathologisch-a na to mische Diagnose: Phlebektasien der Oesophagusvenen. Kein Magengeschwür, kein Duodenalgeschwür. Venöse Blutstauung im kleinen Kreislauf. Keine Mißbildung des Herzens. Foramen ovale und Ductus Botalli geschlossen. Kleine bronchopneumonische Herde in beiden Lungenoberlappen. -

Wir sind also absolut gezwungen, darauf zu verzichten, alle Fälle von Melaena auf eine gemeinsame Genese zurückzuführen. Wir beschränken uns vielmehr darauf, orale oder anale Blutungen bei Neugeborenen oder kleinen Kindern als Melaena zu bezeichnen, wemn das Blut aus dem Verdaungstrakte selbst stammt. Die Bezeichnung 
„Melaena spuria" fällt weg, als Widerspruch in sich selbst. Und die Epitheta „vera" und ,idiopathica" haben keinen Sinn mehr. Wir bleiben uns dabei aber bewußt, daß wir ein Symptom verschiedener Genese mit demselben Namen belegen, und daß die Melaena der Kinder nichts Wesensversehiedenes von den Blutungen der Erwachsenen aus dem Darmtraktus ist. Wir haben die gleiche Bezeichnung für das Symptom, ob ihm ein anatomisches Substrat zugrunde liegt, wie in den 6 zuerst angeführten Fällen, oder ob keinerlei Läsionen des Magendarmtraktus festzustellen sind, wie etwa in dem folgenden Fall.

8. S.-Nr. 398/1918. Ch. N., Mädchen, 17 Wochen alt (Zwilling).

Klinische Diagnose: Lebensschwäche. Dünner Stuhl mit Unterbrechungen, teils Mnttermilch, teils Eiweißmilch. Hat eine Viertelstunde vor dem Tode schwarz erbrochen. Melaena.

Pa thologisch-a na to mische Diagnose: Hämatinhaltige Massen im Magen. Anämie, Pädatrophie. Keine Ulceration im Magen oder Duodenum. Keine Oesophagusvaricen. Keine Anzeichen für Lues oder Tuberkulose. Die Magenwand war ziemlich stark, schmierig verändert, so daß eine Mikroskopierung wenig Aussicht auf bessere Beurteilung bot. Tatsächlich erwies das histologische Bild keinen anderen Befund. An einzelnen tieferen Stellen konnten Blutaustritte ins muköse und submuköse Gewebe gesehen werden, die als Diapedesisblutungen gedeutet wurden.

Wo Blutaustritte ohne sinnfällige Veränderung der Gefäßwand oder in der chemisch-physikalischen Beziehung zwischen Blut und Gefäßwand stattgehabt haben, muB man im Blut selbst das auslösende Moment suchen. Hier ist es das Nächstliegende, an eine Steigerung des Blutdruckes zu denken. Wird doch mit der Geburt ein Umschwung in den Zirkulationsverhältnissen hervorgerufen, wie im späteren Leben nie wieder. Vollzieht sich dieser Umschwung sehr plötzlich, setzt also der Abfluß aus der Arteria hypogastrica eher aus, als der Organismus die Arterialisierung des Blutes selbständig in die Wege zu leiten vermag, so kann nach Bra un schon hierin die Ursache für die Melaena erblickt werden. Wiederhofer dentet in solchem Sinne darauf hin, daß die Blutzufuhr zu den Bauchorganen in gleichem Maße bestehenblciben, während die Druckentlastung durch die Nabelgefäße plötzlich in Fortfall kommen kann. Auch Ritter teilt der Stauung bei der Geburt eine wesentliche Rolle bei der Entstehung der Melaena zu. Noch gesteigert werden die Anforderungen an das Gefäßsystem Zeitschrift für Kinderheilkunde. 0 . XXIII. 
bei Schwergeburten, besonders dann, wenn die Zufuhr durch Kompression der Nabelschnur erschwert oder unmöglich gemacht wird. Es fehlt denn auch nicht an Autoren, die einer solchen Kompression der Nabelschnur die Schuld an der Entstehung eines ,Schleimhautkatarrhs" und schließlich des Blutabgangs aus Mund oder After geben. Es gehören zu ihnen sowohl diejenigen, die in zu früher (Wiederhofer, Ki wisch, Chotowitzky), als auch diejenigen, die in zu später Abnabelung (Pora k) das ätiologische Moment sehen. Wird die Nabelschnur komprimiert, so können Thromben entstehen, die nach Eiselsberg und Beneke durch das Pfortadersystem auf venösem Wege in die Magenwand kommen und dort Erosionen und Ulcus mit folgendem Bluterbrechen auslösen sollen. An der Angabe, daß der Ductus Arantii als Thrombenpassage diene (Pomorsky, Betz), müssen wir wegen der Enge dieses Gebildes zweifeln. Nach v. Franqué soll bereits heftiges Schreien und forcierte Atembewegung die Verschleppung eines Thrombus in die Magenschleimhaut bewerkstelligen können. Auch auf arteriellem Wege soll nach Landa u bei Offenbleiben des Ductus Botalli der gleiche Vorgang möglich sein. Wir erkennen in solchen Theorien das gleiche heiße Bestreben, wie beim Ulcus ventriculi, eine genetische Erklärung unter Heranziehung der behinderten Hämodynamik zu finden.

Auch sonst wurden Anomalien des GefäBsystems öfter bei Melaena beobachtet und als ursächlicher Faktor bezichtigt. Reuss gibt an, die Kombination der Melaena mit Störung der Blutverteilung wegen Herzfehlers sei häufig. Gefäßanomalien sind von Betz und Meberding bei Melaenakindem beobachtet worden. Namentlich soll auch ein kleines kongenitales Aneurysma der Arteria pancreatico-duodenalis leicht Veranlassung zu Blutungen in -die Magenschleimhaut geben können. Ferner ist daran gedacht worden, daß eine von Schmorl beschriebene, fettige Gefäßdegeneration - vorausgesetzt, daß sie keine im Gefolge der Melaena auftretende Erscheinung ist - Blutaustritt verursachen möchte.

Bekanntlich führen lange nicht alle peptischen Erosionen oder Ulcera zu Bluterbrechen oder Blutstühlen, wenn sie auch meistens mit minimalen Intestinalblutungen verbunden sein mögen, mit jenen okkulten Blutungen, die recht leicht übersehen werden können. Hiermit stimmen statistische Erhebungen überein, die Chzanowskys Behauptung, Uleus sei wesentlich häufiger als Melaena, vollauf bestätigen. Diese stellt sich, wie aus folgenden Zahlen hervorgeht, in 
ca. 1 pro Mille der Geburten ein; denn die Melaena wurde beobachtet von Unger bei 9 Kindern von 6000 Geburten, von Hecker und Buhl bei 8 von 4000 , von Silberman $n$ bei 37 von 29333 , von Shukowsky bei 1 von $5-800$ und von Baiseh bei 14 von 14390 . Erinnern wir uns der Sektionserfahrungen, daß das kindliche Magengeschwür häufiger gefunden wird ohne die Stigmen der Darmblutung als mit solchen, oder gar als Melaenafälle ohne anatomischen Ausdruck, so können wir Chzanowsky nur beipflichten.

Neben den peptischen Affektionen auf Grund von Zirkulationsstörungen können auch durch bakteriämische Metastasenbildung im Magendarmtraktus Geschwüre entstehen, die zu Melaena führen. Andererseits kann bei Infektionskrankheiten auch ohne jeden Gewebsdefekt Melaena zustande kommen. Bene ke erinnert mit Recht an die Parallele, die sich hier mit dem Vomito negro bei Gelbfieber ergibt. Die von Wiederhofer mitgeteilten Fälle von Pyämie der Nabelgefäße stehen wohl auf der Grenze zwischen der Melaena nach Sepsis und der nach Gefäßveränderungen, da ihnen wahrscheinlich septische Embolien zugrunde liegen. Vielleicht wäre daran zu denken, daß in diesem Falle die Melaena sowohl durch die Folgen der Ischämie veranla Bt, als vor allem durch eine toxisch gesteigerte Durchlässigkeit der Gefäßwand hervorgerufen werden könnte. Kann doch auch durch Lues neben punktförmigen Hämorrhagien der äußeren Haut Melaena des Magendarmtraktus entstehen. Shukowsky hat unter 25 Melaenafällen 3 mit Lues behaftete gesehen; derselbe Autor läßt aber nicht nnerwähnt, daß die oft mit Lues verbundene Lebercirrhose oder daß Hemmungsbildungen des Herzens auf dem Wege der Stauung Melaena verursachen können. Hier sei auch an den vorletzten obenerwähnten Fall erinnert. Solche Melaenafälle wären also zu den Affektionen zu rechnen, die durch Zirkulationsstörungen hervorgerufen wurden.

Immerhin ist bekannt, daß es krankhafte Zustände, wie die Purpura, gibt, bei denen die Gefäße ohne einen uns ersichtlichen. Grund Blut durchlassen. Es ist auch schon vor langer Zeit von Henoch darauf hingewiesen worden, da $\beta$ die bei Kindern beobachteten Formen der Purpura sich durch intestinale Blutungen auszeichnen. Für die Pur* pura selbst wurde nun von manchen ein bis jetzt unbekannter Erreger angenommen. Man war versucht, auch an einen Melaenaerreger in Gestalt eines Mikroorganismus zu denken. Zu dieser tberlegung führte besonders das Vorkommen von Fällen, in denen bei Zwillingen (Rillet) oder aber auch bei Mutter und Kind (Betz) zur selben Zeit 
Melaena aufgetreten war. Die Deutung solcher Beobachtung im infektiösen Sinn ist aber sehr anfechtbar. Kann man doch gerade die eben genannten Fälle als Stütze für die Anschauung auffassen, daß die Melaena, wie es auch von manchen für die Purpura angenommen wird, nur die Folge bzw. eine Ausdrucksform konstitutioneller Minderwertigkeit ist, welche unter dem Bild der hämorrhagisehen Diathєse für uns sinnenfällig wird. In dieser Hinsicht sei erwähnt, daß Betz den Fall zweier Schwestern mitteilt, welche von Melaena befallen wurden, daß ferner Franq ué dasselbe bei zwei aufeinanderfolgenden Kindern einer Mutter beobachten konnte. Jedesmal waren Mutter und Kinder aus einer Familie, die dadurch aufgefallen war, daß mancherlei Mißbildungen bei ihren Mitgliedern bemerkbar geworden. Mit der Annahme einer konstitutionellen Basis für das Zustandekommen der Melaena stimmt es auch überein, wenn man heute mehr und mehr dazu neigt, Blutungsstigmata, Purpura und ähnliche Erscheinungen auf eine neurogene Basis zurückzuführen. Mora wi tz hat berichtet, daß gerade Purpuraerscheinungen mit Neuralgie und Herpes zoster in Zusammenhang stehen können.

Ist das richtig, so gewinnen wir damit die Möglichkeit. Purpura, Melaena und peptische Affektionen unter einem genetischen Gesichtswinkel zu betrachten. Für die peptischen Affektionen kann man heute, wie oben erwähnt wurde, neben anderen Entstehungsmöglichkeiten gerade vagotonischen und angiospastischen Störungen eine häufige Mitwirkung zuerkennen. Nun gehen die Ulcera peptica ursprünglich recht oft aus Blutungsstellen der Schleimhant, aus ,Stigmen“, wie Bene ke sie benennt, hervor. Solche Stigmen können bestimnt experimentell erzeugt werden. Diese Experimente können also auch als Stütze der Auffassung einer neurogenen Entstehung der Melaena dienen. Man denke nur daran, daß Pomorsky Magenwandblutungen durch Reizung bestimmter Hirnteile, insbesondere des Vasomotorenzentrums. hervorgebracht hat. Es sei weiter noch darauf hingewiesen, dab Beneke durch verschiedenartige, den nervösen Apparat des Magens irritierende Einwirkungen punktförmige Blutaustritte der Magenschleimhaut, ,Stigmata“, erzeugt hat; anderen Autoren (Kobajashi, Schmincke) ist Ähnliches geglückt.

So wird nun auch verständlich, daß schwere Geburten, die mit Druck auf den Schädel einhergehen und leicht zu Störungen in der. Blutverteilung des Gehirıs und der Himhäute führen - man denke an die Tentoriumblutungen! -, zu Melaena, als Symptom einer sich 
im Magendarmwandbereich auswirkenden nervösen Störung, disponieren können. Allgemein läßt sich das an Hand der Literatur nicht nachweisen, denn in den großen Sammelberichten von Vassmer und Theile fallen Schwergeburten nicht besonders oft mit Melaena zusammen. Theile gibt 3 Fälle unter 64 Geburten, Vass mer 4 unter 67 an, jedoch ist zu bedenken, daß häufig die Katamnese gar nicht erhoben worden ist. Beneke hat jedenfalls das gemeinsame Vorkommen von Melaena neonatorum aus Magenschleimhauterosionen bei Residuen ausgedehnter Tentoriumzerreißungen mit meningealer Blutung in kausale Beziehung gebracht, und zwar in dem Sinne, daß die Magenerosionen der Hirnirritation gefolgt seien. Für eine solche neurogene Auffassung spricht auch die Beobachtung von Sochaczewsky, der bei einem meningitischen, 10 Monate alten Kinde Blutstühle sah. Hier möchte ich auch noch einmal an den oben mitgeteilten 3. Fall erinnern. Wenn Buhl auf die Ähnlichkeit der Melaena mit Phosphor-, Arsen- und Antimonvergiftungen hinweist, so sei daran erinnert, daß bei Phosphorintoxikationen zuerst die Gehirnzellen ihre Funktion einstellen, und daß Arsen und Antimon zentral und besonders auf Blutcapillaren des Darmes lähmend wirken (Me yer-Gottlieb). Und wenn Rilliet und Barthet von einer Atonie der GefäBe bei Melaena sprechen, so bekennen sie sich damit zu der Auffassung, daß eine neurovasculäre Störung hier von Bedeutung sei.

Aus all diesen Betrachtungen resultiert also der Eindruck, daß bei Innervationsstörungen der Gefäße Ulcus mit oder ohne Blutung oder aber - darauf wies besonders Bene ke hin - Melaena allein entstehen kann. Ein einheitlicher Entstehungsmodus wird wohl nicht vorliegen. Wie für das Magengeschwür, so werden auch hier mehrere Bedingungskomplexe genetisch als möglich berücksichtigt werden müssen. Bietet die neurogene Theorie einesteils in Verbindung mit der konstitutionspathologischen Anschauungsweise, andererseits mit der traumatisch-irritativen Handhabe zur Deutung mancher Beobachtungen, so darf man nicht versäumen, in einschlägigen klinischen Fällen nach anderweitigen Stigmen zu fahnden oder die Möglichkeiten traumatischer, cerebraler bzw. zentralnervöser Einwirkung ohne Uberlegung sonstiger ätiologischer Bedingungskomplexe festzulegen, d. h. wenn es sich um Todesfälle handelt, durch sachgemäße Sektionen die Unterlagen für die Gewinnung brauchbarer Anhaltspunkte für die verschiedenen Entstehungsmöglichkeiten zu schaffen. Namentlich ist es auch von Interesse, solchen Vorkommnissen vom 
Standpunkte der Vererbungsfrage näherzutreten. DaB vom Standpunkt der konstitutionellen Pathologie hier eine verheißungsvolle Arbeit vorliegt, zeigt $u$. a. eine Arbeit von Hirsch und Schneider. Diese Autoren haben bei einem an Bronchotetanie verstorbenen Kind. in der Ileocöcalgegend ein Geschwür gefunden, das ihrer Ansicht nach durch lokale Gefäßkrämpfe als ischämisch geschädigte Schleimhautstelle in Form einer durch Bakterienwirkung komplizierten Nekrose entstanden war. Vielleicht nehmen weitere Beobachter der Melaena auch auf das Verhältnis zwischen Pylorospasmus und Melaena Rücksicht, denn es wäre möglich, daß auch hier Brücken bestehen könnten.

Betrachten wir endlich die Melaena lediglich als eine symptomatische Erscheinung, welche quantitativ minimal, andererseits abundant sein kann, bedenkt man ferner, daß diese Blutungen ein Hauptzeichen der peptischen Affektion sind, so resultiert daraus, daß es billig ist, die Frage der Melaena der Frage des peptischen Geschwürs unterzuordnen. Daß das Vorkommen peptischer Affekte im Kindesalter sowohl für Magen als Duodenum nichts Seltenes ist, wurde schon oben betont. Immerhin mag ihre Häufigkeit noch größer sein, als wir heute amehmen, denn, wie unsere obenerwähnten Mitteilungen lehren, können solche Affekte wohl überwunden werden, können selbst in so ungünstigem Gelände, wie es das Duodenum darstellt, peptische Läsionen unter Narbenbildung ausheilen.

\section{Literaturverzeichnis.}

Baginsky, Lehrbuch der Kinderkrankheiten (Kapitel über Melaena neonatorum). Baisch, Melaena neonatorum in Winkels Handbuch der Geburtshilfe III. Band. 3. Teil, S. 254. Wiesbaden 1907.

Ba uer, Zur Ätiologie der Melaena neonatorum. Münch. med. Wochenschr. 1904, S. 1207 .

Bech thold, Jahrb. d. Kinderheilk. 1904, S. 347.

Bed mar, Krankheiten der Neugeborenen und der Säuglinge. Wien 1850. S. 75. Bender; Melaena neonatorum. Inaug.-Diss. Freiburg 1907; ref. i. Archiv f. Kinderheilk. 49.

Beneke, Über hämorrhagische Erosionen des Magens. Verhandlungen der Deutschen pathologischen Gesellsch. Kiel 1908. S. 284.

-_ Über Tentoriumzerreißungen bei der Geburt. Münch. med. Wochenschr. 1910, Nr. 41.

v. Berg mann, Ulcus duodeni und vegetatives Nervensystem. Berl. klin. Wochenschrift 1918, Nr. 51.

- Das spasmogene Ulcus pepticum. Münch. med. Wochenschr. 1913, Nr. 4.

Betz, Zur Ätiologie der Melaena neonatorum. Virchow-Hirschs Jahrb. 2. 1879. 
Binz, Perforierendes Magengeschwür beim Neugeborenen. Berliner klin. Wochenschrift 1865.

Bisset, Lancet 1905.

Blühdorn, Therapia sog. unstillbarer Blutungen im Säuglingsalter. Berliner klin. Wochenschr. 1913, Heft 1.

Borla nd, H. H., Infantile acute eccema; haematemesis; duodenal ulcer. Lancet 1913.

v. Chrzanowski, Zwei Falle von Melaena. Archiv f. Kinderheilk. 21, 321. 1896/97.

- Ulcus duodeni et ventriculi. Ebendort.

Dietrich, H. A., Statistische und ätiologische Bemerkungen zu Ulcus pepticum duodeni. Münch. med. Wochenschr. 1912. Nr. 12.

Ebstein, Experimentelle Untersuchung über das Zustandekommen der Blutextravasate in die Magenschleimhant. Arehiv f. experim. Pathol. u. Pharmakol. z, 183 .

Fischer, L. (jun.), Zur Kasuistik und Ätiologie der Melaena neonatorum. Münch. med. Wochenschr. 1897. S. 304.

Fi schl, Lokale Erkrankungen des Magens usw. In Pfaundler-Schlossmanns Handb. Bd. 3, S. 148.

Fleischmann, Zur Ätiologie der Melaena vera. Jahrb. d. Kinderheilk., N. F., 3, H. 2, S. 211.1870.

Flesch, Zur Diagnose und Pathogenese des duoden. Gesehwürs im Säuglingsalter. Jahrb. d. Kinderheilk. 1912, S. 542.

v. Franqu é, Über tödlichen Affekt der Magenschleimhaut nebst Bemerkung. zur Melaena. Beiträge z. Geburtsh. u. Gynäkol. 10, 187. 1907.

Finkelstein in Feers Lelırbuch der Kinderheilkunde. Jena 1912. S. 125.

Gärtner, Über Melaena neonatorum. Zentralbl, f. Gynäkol. 18, 691. 1894.

Genell, Melaena neonatorum. Brit. med. Journ. 1, 18.1896.

Gruber, Georg B., Zur Statistik des peptischen Affektes im Magen, Oesophagus und Duodenum. Münch. med. Wochenschr. 1911, Nr. 31 und 32.

- Zur Lehre über das peptisehe Duodenalgeschwür. Mitt. a. d. Grenzgeb. d. Med. u. Chir, 25, H. 3, S. 465. 1912.

- Zur Frage über das Zustandekommen des peptischen Magen- und Duodenalgeschwürs. Deutsches Archiv f. klin. Chir. 110. 1913.

- und Lanz, Ichaemische Herzmuskelnekrose bei einem Epileptiker nach Tod im Anfall. Archiv f. Psych.; erscheint demnächst.

Gruber, Gg. B., Über das Zustandekommen des peptischen Gesehwürs. Münchener mediz. Woch. 1919.

Gut man n, Zur Behandlung der Meluena neonatorum. Therapeut. Monatsh. 1899, S. 552.

Hecker und Buhl, Klinik d. Geburtsk. 2. 1864.

Henoch, Magengechwüre bei Kindern usw. In Vorl. i. Kinderkrankh. S. 59. He ubner, Kapitel über Melaena im Lehrb. d. Kinderheilk.

Hirseh und Schneider, Dünndarmgeschwür als Erscheinungsform der Spasmophilie. Monatsschr. f. Kinderheilk. 15, H. 3. 1918.

Josselin, zit. nach Micha ud.

Katzenstein, Beiträge zur experimentellen Entstehung des Magengeschwürs. Archiv f. klin. Chir. 101, 1. 1913. 
Kiwisch, zit. nach Simmonds.

Kling, Uber Melaena neonatorum. Inaug.-Diss. München 1875.

Knö pfel macher in Pfaundler-Schlossmanns Handb. Bd. 1, S. 389.

Kobayashi, Über experimentelle Erzeugung von peptischen Erosionen. Frankf.

Zeitschr. f. Pathol. 3, 566. 1909.

Küttner, Uleus duod. Archiv f. klin. Chir. 101, H. 2.

Langerhans, Über Melaena traumatica. Ärztl. Sachverst.-Ztg. 2, 2. 1896.

Leu be in Ziemssens Handb. d. spez. Path. u. Ther. Bd. 7, II, S. 144. 1874.

Lichtenbelt, Die Ursachen des chronischen Magengeschwürs. Jena 1912.

Marchand in Marchand-Krehls Handb. d. allg. Pathol. Bd. 2, Abt. I, S. 299.

Meberding, Über Melaena neonatorum. Vortr. i. d. Med. physik. Gesellech. zu

Würzburg 1888.

Melchior, Chirurgie des Duodenums $=$ Neue deutsche Chir. 25, $77 \mathrm{ff} .1917$.

- und Löser, Ergebnisse der Chirurgie und Orthopädie 2, Nr. 7-9. 1911.

Meyer-Gottlieb, Experimentelle Pharmakologie. Berlin u. Wien 1911.

Micha ud in Mohr-Staehelins Handb. d. inn. Med. Bd. 3, Abt. II.

Möller, Die Pathologie des Ulcus usw. Erg. d. inn. Med. u. Kinderheilk. જ, 569.

Mo nro, Case of Melaena neonatorum usw. Brit. med. Journ. 1, 1501. 1896.

Morawitz in Mohr-Staehelins Handb. Bd. 4.

Morgan, A case of Melaena neonatorum. Brit. med. Journ. 2, 1294. 1895.

M ueller, Zur Kasuistik u. Theorie der Melaena neonatorum. Münch. med. Wochenschrift 1894, S. 245.

Neumann, Fall von Melaena neonatorum mit Bemerkungen über hämorrhagische Diathese Neugeborener. Archiv f. Kinderheilk. 12, 54. 1890/91.

Nicholson, Rep. of a case of Melaena neon. due apparently to an infection by the Bac. pyocyaneus. Amer. Journ. of med. science Philad. u. N.-York 120, 417. 1900 .

Oberndorfer, zit. n. G. B. Gruber.

Pomorsky, Experimentelles zur Ätiologie der Melaena neonatorum. Archiv f. Kinderheilk. 14, $165.1891 / 92$.

- Zur Ätiologie der Mel. vera. Deutsche med. Wochenschr. 1888, S. 762.

v. Preuschen, Die Läsion des Zentralnervensystems bei der Geburt als Ursache der Melaena neonatorum. Zentralbl. f. Gynäkol. 18, 201. 1894.

Rheiner, Über Melaena neonatorum. Korresponden̂zbl. f. Schweiz. Ärzte 28, 524. 1898.

v. Reuss, Die Krankheiten der Neugeborenen. Berlin 1914.

Rilliet und Barthet, Handb. d. Kinderkrankh. Bd. 2, S. 371 und Bd. 3, S. 295.

Roether, Ule. duod. im Säuglingsalter. Inaug.-Diss. Greifswald 1916.

Rössle, Das runde Geschwür des Magens usw. Mitt. a. d. Grenzgeb. d. Med. u. Chir. 25, H. 4, S. 765.1912.

- Münch. med. Wochenschr. 1912, Nr. 22.

Rüti ma yer, Über die geographische Verbreitung und Diagnose d. Ulc. ventriculi rot. Wiesbaden 1906.

v. Rundstedt, Über Melaena neonatorum. Archiv f. Gynäkol. 89, 105 u. 275. 1909.

Schmid-Mommard, Über einen genesenen Fall von Melaena neonatorum. Münch. med. Wochensehr. 1892, S. 47. 
Schminke, Über experimentelle Erzeugung sog. peptischer Erosionen des Magens bei Kaninchen usw. Phys.-med. Gesellsch. Würzburg 1910.

Sch morl, Fünf Fälle von Melaena. Ref. i. Monatsschr. f. Geburtsh. u. Gynäkol. 1901.

Schütze, Ein Fall von Melaena neonatorum. Zentralbl. f. Gynäkol. 1894, S. 207.

Shukowsky, Archiv f. Kinderheilk. 43. 1907.

Simmonds, Zur Ätiologie der Melaena neonatorum. Jahrb. d. Hamb. Staatskrankenanst. 1890. Leipzig 1892, Bd. 2, S. 484.

Sochaczewsky, Zur Pathogenese der Darmblutungen im Säuglingsalter. Archiv f. Kinderheilk. 50. 1909.

Spiegelberg, Ein Fall von Melaena neonatorum mit außergewöhnlichem Sitz der Blutungsquelle. Prager med. Wochenschr. 23, 61. 1898.

Staehelin in Mohr-Staehelins Handb. Bd. 3, Abt. I, S. 691.

Stroh me yer, Die Pathogen. d. Ule. ventr. usw. Beiträge z. pathol. Anat. u. allg. Path. 54. 1912.

Swoboda, Gibt's eine Melaena vera? Prager med. Wochenschr. 25, 581. 1900.

- Zur Ätiologie der Melaena. Wiener klin. Wochenschr. 1896, S. 916.

Theile, Über Geschwürsbildung des Gastro-Duodenaltraktus im Kindesalter. Erg. d. inn. Med. u. Kinderheilk. 16, 302. 1919.

Thie m, Handb. d. Unfallkrankh. 2. Aufl. Bd. 2, Abt. II, S. 439.

Tross, Über einen Fall von Melaena neonatorum. Deutsohe med. Wochenschr. 14, S. 433.1888.

Uffen heimer, Über den Säuglingsmagen usw. Erg. d. inn. Med. u. Kinderheilk. 2. 1908.

Umber, Melaena neonatorum. In Mohr-Staehelins Handb.

Unger, Melaena vera. Wiener klin. Wochenschr. 1912.

Vass mer, Über Melaena neonatorum. Archiv f. Gynäkol. 89, 275. 1909.

Veit, Fall von Melaena neonatorum. Zeitschr. f. klin. Med. 4, 471. 1882.

Vogt, Berliner klin. Wochenschr. 50, 941. 1913.

Wiederhofer in Gerhardts Handb. d. Kinderkrankh. 4, 399. 1880.

v. Zezschwitz, Über einen Fall von Melaena vera. Münch. med. Wochenschr. 1888 , S. 483 u. 504.

Ziegler, Lehrb. d. spez. pathologischen Anatomie. 